\title{
Caracterización de lesiones de causa externa asociada a violencia en jóvenes entre 14 a 26 años en un Hospital Público de tercer nivel de la ciudad de Cali entre los años 2012 a 2014
}

\author{
${ }^{1}$ Karyna Reyes Caicedo, ${ }^{2}$ Bryan Steven Urrea Trochez, ${ }^{3}$ María Juliana Chaves, ${ }^{4}$ Manuela Escobar \\ ${ }^{5}$ Mónica A Morales García, ${ }^{6}$ Marisol Badiel, ${ }^{7}$ Carlos A Ordoñez
}

\begin{abstract}
RESUMEN
Introducción: La violencia juvenil se ha clasificado como un problema de salud pública a nivel mundial debido a su alta incidencia, elevados costos al sistema de salud y a la sociedad en términos de años de vida perdida. A partir del año 2012 se implementa una política pública para disminuir los índices de violencia juvenil, de la que no se han evaluado los efectos desde el orden de la atención en salud.
\end{abstract}

Objetivo: Describir las características de la violencia juvenil en el período 2012-2014 en un hospital púbico de nivel I en Cali-Colombia después de que las políticas públicas fueron implementadas en dicha ciudad.

Materiales y métodos: Estudio de cohorte retrospectiva. A partir del Registro Internacional de (ITR/SPT-ITSDP) Trauma del Hospital Universitario del Valle, se incluyeron todos los pacientes consecutivos entre 14-26 años que ingresaron con alguna lesión de causa externa asociada a violencia.

Resultados: Del Registro Internacional, el 32\% (7715/24084) se reportaron como lesión secundaria a trauma entre los 14-26 años, y de estos el 37.33\% (2880/7715) estaban asociados a violencia. Más frecuentemente se presentaron casos entre 14-18 años en el año 2012 y para los años 2013 y 2014 , en el grupo de los 19 a 22 años. El principal tipo de trauma fue el penetrante (81.2\%) por arma de fuego (54.1\%), seguido las heridas por arma blanca (25.2\%). En el $17,9 \%$ se encontró consumo de alcohol concomitante, y el $29,8 \%$ con uso de sustancias psicoactivas. La tasa de violencia juvenil

${ }^{1}$ Estudiante de Medicina (5to año), ${ }^{2,3}$ Estudiante de Medicina (6to año), ${ }^{4}$ Estudiante de medicina (3er año), ${ }^{5}$ Estadística ${ }^{6}$ Profesor Titular, Doctora en Epidemiología, ${ }^{7}$ Profesor Titular y Cirujano de Trauma y Emergencias

${ }^{1-4}$ Universidad del Valle, Facultad de Salud - Grupo de investigación Hospital Universitario Del Valle, Cali, Colombia

${ }^{5}$ Grupo de investigación Hospital Universitario del Valle, Cali Colombia

${ }^{6}$ Grupo Epidemiología del Trauma y Lesiones, Universidad del Valle, GRIEPIS, Universidad Libre - Seccional Cali, Colombia

${ }^{7}$ Intensivista, Fundación Valle del Lili. Director Departamento de Cirugía, Facultad de Salud, Universidad del Valle, Hospital Universitario del Valle, Cali, Colombia

Corresponding Author: Karyna Reyes Caicedo, Estudiante de Medicina (5to año), Universidad del Valle, Facultad de Salud Grupo de investigación Hospital Universitario Del Valle, Cali Colombia, e-mail: karinareyesc20@hotmail.com presentó cambios significativos de $55.7 \%$ en el 2012 a $37.8 \%$ en el 2014, $p<0.0001$ ) y que repercute en la disminución en la tasa de mortalidad global de $7.4 \%$ a $3.3 \%, p=0.0349$ respectivamente.

Conclusiones: En la población entre 14-26 años las lesiones por trauma entre los años 2012-2014 se presentó una disminución significativa en la incidencia anual $(p<0.0001)$ y disminución en la tasa de mortalidad global. Al parecer la tendencia observada puede ser el reflejo del impacto de la implementación de la política pública.

Palabras Clave: Arma de fuego, Joven, Mortalidad juvenil, Política pública, Trauma, Violencia.

\section{ABSTRACT}

Introduction: Youth violence has been classified as a public health problem worldwide, due to its high incidence, high costs to health system and society in term of lost life years. Since 2012 a public policy has been implemented to decrease youth violence index, but the results have not been assessed from the perspective of health care.

Objective: To describe the characterstics of youth violence in the period 2012 to 2014, in a public hospital level I of Cali, Colombia, after the implementation of a public policy.

Materials and methods: Retrospective cohorts study. From International Trauma Register of Hospital Universitario del Valle, all consecutive patients between 14 to 26 years who entered with any external cause injury associated with violence, were included for study.

Results: From the International Trauma Register, 32\% (7715/24084) cases were reported as trauma injury in patients between 14 to 26 years old, and of these, $37.33 \%$ (2880/7715) were associated with violence. During 2012, cases were more frequently reported in the age group of 14 to18 years, and in 2013 and 2014, in 19 to 22 years age group. The main trauma type was penetrating (81.2\%): firearm $(54.1 \%)$ and thenwhite weapon $(25.2 \%)$. There was alcohol consumption on $17.9 \%$, and $29.8 \%$ of psychoactive substances. Youth violence rate changed significantly from $55.7 \%$ in 2012 to $37.8 \%$ in 2014 , $(p<0.0001)$, and this resulted in the decrease of global mortality rate from $7.4 \%$ to $3.3 \%, p=0.0349$ respectively.

Conclusion: Population between 14 to 26 years old had a significant decrease in annual incidence of trauma injuries between 2012 and $2014(p<0.0001)$ and a decrease in global mortality rate. Apparently, the observed trend could be explained by the impact generated due to the implementation of the public policy. 
Keywords: Firearm, Public policy, Violence, Youth, Youth mortality.

How to cite this article: Caicedo KR, Trochez BSU, Cháves MJ, Escobar M, García MAM, Badiel M, Ordoñez CA. Caracterización de lesiones de causa externa asociada a violencia en jóvenes entre 14 a 26 años en un Hospital Público de tercer nivel de la ciudad de Cali entre los años 2012 a 2014. Panam J Trauma Crit Care Emerg Surg 2018;7(1):4-9.

\section{Source of support: Nil}

\section{Conflict of interest: None}

\section{INTRODUCCION}

La Organización Mundial de la Salud define violencia como "El uso deliberado de la fuerza física o el poder, ya sea en grado de amenaza o efectivo, contra uno mismo, otra persona o un grupo o comunidad, que cause o tenga muchas probabilidades de causar lesiones, muerte, daños psicológicos, trastornos del desarrollo o privaciones." ${ }^{\text {"1 Se }}$ considera además, como violencia juvenil cuando esta es perpetuada en el grupo etario de jóvenes y adolescentes, es decir, en la población que se encuentra entre los 10 y los 24 años. ${ }^{2}$ La violencia ha sido parte siempre de la experiencia humana; no obstante, en las últimas décadas su impacto ha sido tan importante que en 1996 se declaró, por la asamblea mundial de la salud, como un problema de salud pública. ${ }^{3}$ La violencia juvenil se encuentra dentro del grupo de violencia interpersonal comunitaria y para efectos de este trabajo, se trabajará la violencia juvenil de naturaleza física, tanto fatal como no fatal, debido a que es allí donde se encuentran las cifras más altas, así como las mayores pérdidas al sistema.

Cada año, según cifras de la OMS, más de 1,4 millones de personas pierden la vida debido a la violencia, y de esta cifra, alrededor de 200000 muertes se producen por homicidio en personas entre los 10 y los 29 años (diariamente mueren 565 jóvenes a causa de la violencia interpersonal ${ }^{4}$ Así, el homicidio se constituye como la cuarta causa de muerte a nivel mundial en jóvenes. ${ }^{5}$

Debido a la importancia de este fenómeno, se han realizado múltiples estudios que han identificado como factores de riesgo ser hombre, estratos socioeconómicos bajos, consumo de cigarrillo y SPA, baja autoestima, baja escolaridad entre otras. ${ }^{6}$ No obstante, según el informe sobre la situación de la prevención mundial de la violencia 2014, expone que menos de la mitad de los países encuestados recogen información acerca de la violencia juvenil (solo lo hace el 26\% de los 133 países encuestados), pero se encuentra que el 53\% de los países participantes tienen planes de acción nacionales para prevenir y tratar la violencia juvenil, lo que sugiere que gran parte de la planificación y el establecimiento de normas se lleva a cabo sin datos. ${ }^{7}$

Añadido a la problemática de la falta de datos para la implementación de estrategias adecuadas, se encuentra que la violencia juvenil, no solo aporta altos índices de mortalidad y coste humano y social inmediato, sino también elevadas pérdidas económicas al sistema, que se pueden evidenciar al revisar los indicadores Años de Vida Saludables Perdidos AVISAS ${ }^{7}$ En Colombia, para el año 2014 se encontraron unos años de vida potencialmente perdidos correspondientes a 900.878 años, y una pérdida de 371.142 años de vida saludables por lesiones no fatales, cifras dadas en su mayor parte, por lesiones dentro de la población juvenil. Para Latinoamérica, al estimar el costo de oportunidad de homicidios de la región, el detrimento al capital ex aproximadamente de 27,737 millones de dólares por año, ${ }^{8}$ perdiendo entonces alrededor del 14\% del Producto Interno Bruto por violencia. Se toman en cuenta los datos de la región debido a la carencia de más datos nacionales.

Así, la caracterización del comportamiento de la violencia que se presenta en la población joven, así como de las lesiones que esta produce, se constituyen como datos valiosos e importantes, pues aportan bases sólidas que describen los patrones de las diferentes variables modificables a través de diversas estrategias para controlar los índices de violencia juvenil. A través de este trabajo pretendemos evaluar la tendencia de violencia juvenil interpersonal en los últimos años en la ciudad de Cali, y establecer posibles inferencias acerca de la efectividad de las políticas públicas dirigidas a esta población que se han implementado para el período de tiempo a estudio.

\section{MATERIALES Y METODOS}

\section{Diseño}

Es un estudio descriptivo, tipo cohorte retrospectiva que a partir del Registro Internacional de Trauma de la Sociedad Panamericana de Trauma mediante el Programa Internacional de Sistemas de Trauma (ITR/SPT-ITSDP) instalado en el Hospital Universitario del Valle "Evaristo García" E.S.E. Se utilizaron todos los registros de jóvenes entre los 16-24 años con lesión de causa externa en el periodo enero 2012 a Diciembre 2014, el estudio fue aprobado por el comité de ética en investigación institucional. Previamente se ha descrito la metodología del registro. ${ }^{9}$

\section{Sitio}

El hospital corresponde a uno de carácter público de primer nivel de atención y un importante centro hospitalario de referencia en el suroccidente Colombiano, caracterizado por el manejo de la alta complejidad, de afiliación académica a los programas de salud de la Universidad del Valle con reconocimiento internacional. ${ }^{9}$

\section{Definición de violencia juvenil}

La Organización Mundial de la Salud define violencia juvenil como una serie de actos que van desde la 
intimidación y las riñas al homicidio, pasando por agresiones sexuales y físicas más graves, y que se perpetúan en el grupo etario entre los 10 y 29 años. ${ }^{10}$

\section{Análisis estadístico}

Los datos son exportados a una base de datos en un archivo binario, se realiza el análisis en el software estadístico STATA TM $12^{\circledR}$ (StataCorp, College Station, Texas, USA).

Las variables categóricas se presentan en proporciones y para la comparación de variables el test chi cuadrado, para las variables continuas se estimaron medias y medianas con sus respectivas medidas de dispersión (desviación estándar y los percentiles 25 y 75), para las comparaciones se utilizaron la prueba $\mathrm{T}$ de Student o la prueba de Wilcoxon según su distribución de normalidad. EL grupo de edad de 14-26 años se estratifica en 3 categorias dado que su comportamiento es diferente: de 14-18; de 19-21 y de 22-26 años. Se estimaron incidencias y tasas anuales no ajustadas para mortalidad. Se consideró $\mathrm{p}<0.05$ estadísticamente significativo.

\section{RESULTADOS}

Durante el período 2012-2014 ingresaron al Hospital Universitario del Valle, un total de 24.084 pacientes. Las lesiones entre los 14 a 26 años de edad en jóvenes correspondieron a $7.715(32 \%)$. De estos, 2880/7715 (29,5\%) correspondieron a casos de violencia juvenil, que se presentó en mayor proporción en el intervalo etario de los 19 a los 22 años, con una edad promedio de 20,3 $\pm 3,2$ años. En general el tipo de trauma con mayor frecuencia correspondió al secundario a heridas penetrantes, con el $81,2 \%$ (2338/7715), y el mecanismo más prevalente fue el arma de fuego, que representó el 54,1\% (1559/2338) (Tabla 1).

\section{Presentación clínica del trauma}

En cuanto al tipo de trauma, la mayor proporción corresponde al trauma penetrante $(81,2 \%, \mathrm{n}=2338)$, $\mathrm{y}$
Tabla 1: Distribución de las lesiones de causa externa según mecanismo, tipo y severidad del trauma entre 2012-2014

\begin{tabular}{ll}
\hline Característica & Frecuencia \\
\hline Edad, promedio $\pm \mathrm{DE}^{*}$ & $20,3 \pm 3,2$ \\
Edad, categorías, n, (\%) & \\
14-18 & $993(34.4)$ \\
19-21 & $1057(36.7)$ \\
22-26 & $830(28.9)$ \\
Tipo de trauma, n, (\%) & \\
Contuso & $525,(18.2)$ \\
Otro especificado & $17,(0.6)$ \\
Mecanismo, n, (\%) & \\
Arma de fuego & $1559,(54.1)$ \\
Arma blanca & $727,(25.2)$ \\
Agresión & $396,(13.8)$ \\
ISS al ingreso, Mediana (RIQ**) & $4(1-9)$ \\
Glasgow al ingreso, n, (\%) & \\
3-8 & $348,(12.1)$ \\
9-13 & $110,(3.8)$ \\
14-15 & $2360,(81.9)$ \\
Tasa de mortalidad global & $5,3 \%$ \\
Tasa de mortalidad por intencionalidad & $13,2 \%$ \\
\hline *DE: Desviación estándar; ${ }^{* *}$ RIQ: Rango intercuartílico
\end{tabular}

relacionado al mecanismo primario, el arma de fuego se posiciona con más de la mitad de los casos (54,1\%) (Tabla 2).

La severidad del estado hemodinámico al ingreso del paciente, se obtuvo con la escala de coma de Glasgow, la mayor parte de los lesionados ingresan con un buen nivel de conciencia Glasgow 14-15: 81.9\%. De la totalidad de los casos de violencia juvenil, concomitantemente se informó con consumo de Sustancias Psicoactivas, se obtuvo que la de mayor proporción fue la marihuana. De igual manera la mayor cantidad de consumidores se encontró en el intervalo entre los 19 a 22 años. Esta situación fue más frecuente con consumo de SPA, y uso de arma de fuego, seguido por el arma blanca.

\section{Incidencia y Mortalidad}

El período de juventud se dividió en 3 grupos etarios: 14-18 años, 19-22 años y 23-26 años. De la población

Tabla 2: Incidencia de trauma según grupos de edad y tasas de mortalidad no ajustadas. Período $2012-2014$. Fuente: Registro ITR/SPT-ITSDP

\begin{tabular}{|c|c|c|c|c|c|}
\hline & General & 2012 & 2013 & 2014 & Valor de $p$ \\
\hline \multicolumn{6}{|l|}{ Edad, categorías, $n(\%)$} \\
\hline 14 a 18 & $993(34.4)$ & 448, (37.3) & $276,(31.9)$ & $269,(32.9)$ & \\
\hline 19 a 22 & $1057(36.7)$ & $424,(35.5)$ & $323,(37.4)$ & $310,(37.9)$ & \\
\hline 23 a 26 & $830(28.8)$ & $328,(27.3)$ & $264,(30.5)$ & $238,(29.1)$ & \\
\hline \multicolumn{6}{|l|}{ Incidencia } \\
\hline Trauma juvenil, n (\%) & 7715 (32.03) & $2155,(27.9)$ & $2272,(29.4)$ & $3288,(42.6)$ & \\
\hline Trauma por violencia & $2880(37.3)$ & $1200,(55.7)$ & $863,(38)$ & $817,(24.8)$ & 0.0001 \\
\hline \multicolumn{6}{|l|}{ Mortalidad anual por violencia } \\
\hline Tasa anual & 13.1 & 12.3 & 15.6 & 11.9 & \\
\hline Mortalidad general por año (\%) & 5.3 & 7.4 & 6.4 & 3.3 & \\
\hline
\end{tabular}


estudiada el $34,4 \%$ de los casos $(n=993)$, pertenecía al primer intervalo, el $36,7 \%(\mathrm{n}=1057)$ al segundo intervalo y el $28,8 \%(n=830)$ al tercero. De la totalidad de casos según las edades, la mayor incidencia se encontró, para los tres rangos de edades, durante el año 2012 (Tabla 2).

Al realizar el análisis por los años estudiados, se evidencia que hay una disminución en la incidencia de la violencia juvenil ( $\mathrm{p}<0.0001)$. Así, para el 2012 de la totalidad del trauma juvenil, el 55,7\% ( $\mathrm{n}=1200)$ se consideró como violencia juvenil. Durante este período el $37.3 \%$ de los casos se presentó en las edades comprendidas entre los 14 y los 18 años, seguida por las edades los 19 a 22 años $(35,3 \%, \mathrm{n}=424)$ y finalmente el período de los 23 a 26 años $(27,3 \%$ \%, n = 328). En el año 2013 se presentó una disminución de 337 casos en violencia juvenil; se obtiene la mayor incidencia para el intervalo de los 19 a 22 años $(37,4 \%)$ y se mantiene el comportamiento de la menor incidencia en el rango de los 23 a 26 años (30,5\%). El 2014 continúa con el descenso de la incidencia, obteniendo un total de $24.8 \%$. El mayor porcentaje de casos se presenta en el grupo de los 19 a 22 años $(37,9 \%)$, y el menor porcentaje corresponde al grupo de 23-26 años $(29,1 \%)$, conservando la tendencia para este último intervalo.

Finalmente, y a pesar de que el trauma juvenil aumentó durante el período del estudio, la mortalidad general presentó un descenso.

El trauma por violencia también presentó una disminución estadísticamente significativa, con valores de $55,7 \%, 38,0 \%$ y $24,8 \%$ para cada año ( $p<0.0001)$, que se refleja también en la disminución de la mortalidad por intencionalidad ( $\mathrm{p}=0.0349)$, respectivamente para los años 2012, 2013 y 2014.

\section{DISCUSION}

En la población entre 14-26 años las lesiones por trauma entre los años 201212014 se presentó una disminución significativa en la incidencia anual; de 55.7\% en el año 2012 a 24,8\% en el año 2014 ( $p<0.0001)$ y con repercusión en la disminución de la mortalidad global (De 7.4\% a $3.3 \%, \mathrm{p}=0.0349$ ).

Esta tendencia justamente se presenta cuando la política pública es implementada en la ciudad en el año 2012. La violencia juvenil, como todas las epidemias que se presentan en salud pública, tiene unos factores de riesgo que es importante tener en cuenta al momento de medir la vulnerabilidad de una población. Así, la OMS ha dictaminado una serie de condiciones que hacen a un joven más propenso a la violencia juvenil, entre ellos el ser de género masculino, el consumo de SPA, el tabaquismo, el bajo estrato socioeconómico, entre otros. Al análisis de los factores de riesgo de la población estudiada, de los cuales se lograron medir el consumo de SPA, el tabaquismo, y de manera indirecta el estrato socioeconómico a través de la determinación del régimen de seguridad social, se constató que existe una asociación importante entre los factores de riesgo mencionados y la violencia juvenil.

De igual forma, al comparar el comportamiento de la violencia juvenil de Santiago de Cali con la documentada para Latinoamérica, se encuentran cifras bastante por encima de lo esperado, que ponen a Colombia como como uno de los países más violentos de Latinoamérica. Sin embargo, al comparar Colombia con países como Brasil, en donde la tasa de homicidios juveniles asciende a 16,3 por cada 100mil habitantes en el año 2013, se evidencia que es un país más violento a nivel de la juventud que el nuestro. ${ }^{11}$ A pesar de que se observa una disminución en la incidencia de violencia juvenil, así como de mortalidad por intencionalidad durante los 3 años analizados, las cifras siguen encontrándose por encima del promedio nacional y departamental. Al comparar los resultados obtenidos con los datos nacionales aportados por el estudio FORENSIS, es posible evidenciar que mientras que a nivel nacional la incidencia tuvo un aumento y posteriormente una disminución, en Cali el comportamiento de disminución de casos se mantuvo en los 3 años, pero con cifras siempre elevadas. Sin embargo, el comportamiento en lo relacionado a la intencionalidad y mecanismo de trauma, es similar a lo reportado, puesto que se resaltan en ambos casos como principales mecanismos y tipos de trauma, aquellos asociados a riñas y peleas. No obstante, en este estudio se encontró en primer lugar el trauma penetrante y las armas de fuego, y el reporte nacional establece el trauma y objetos contundentes, situación que sugeriría una gran cantidad de factores en relación a la obtención y disponibilidad de armas de fuego y armas blancas dentro de los jóvenes y adolescentes caleños.

Por otro lado al analizar el comportamiento según los grupos etarios, se observa que existe un comportamiento muy similar al esperado, puesto que la mayor incidencia se encontrada se presenta para el intervalo ente 19 y 22 años, al igual que lo reportado por el estudio FORENSIS, con excepción del año 2012, en donde este estudio halló una incidencia mayor en el grupo de 14 a 18 años.

Según los datos brindados por la Personería de Santiago de Cali en su informe del 2014, se coincide con la principal asociación a riñas, y la mayor incidencia en el grupo etario 19-22 años, que se repiten en nuestra ciudad. Es de importancia resaltar, que la disminución en los casos de violencia juvenil, inicia a partir del año 
2012, un año después de la implementación del Plan Local Juvenil, reglamentado por el decreto 945 de la Alcaldía de Santiago de Cali, en el año 2006. Al analizar las cifras de mortalidad, se observa un comportamiento acorde con el comportamiento de la incidencia de violencia juvenil, un patrón de disminución para los 3 años, cifras que concuerdan con la disminución del promedio nacional. Es de resaltar, que las cifras nacionales de los años evaluados evidencian siempre la mayor cantidad de casos en el rango de los 20 a 24 años, sin embargo en este estudio no se determinó la mortalidad según rangos de edad. De acuerdo a todo lo anterior se puede inferir que la implementación del plan local juvenil ha tenido un impacto positivo sobre la población, puesto que se ha presentado una disminución de los indicadores de violencia, a pesar de que se realizó un estudio en el que se encontraron grandes fallos en su implementación. Sin embargo, a pesar de que el comportamiento está acorde con el nacional, las cifras continúan estando elevadas, en relación al resto del territorio colombiano y latinoamericano.

Se debe recalcar que el tema de la violencia juvenil se ha abordado también desde el aspecto conductual psiquiátrico. Un estudio realizado con dos cohortes de diferentes ciudades europeas seguidas durante 4 años, evidenció que sí hay una asociación lineal entre la depresión en personas jóvenes y resultados violentos posteriores, con un aOR de 2,1 (95\% IC $=1.7-2.7) .{ }^{12}$ Otro estudio en el que se comparó una población de hombres jóvenes chinos con una cohorte del Reino Unido, demostró que la incidencia de la violencia en los Chinos es menor que en los Británicos (AOR 0.59, 95\% CI 0.48-0.72, $\mathrm{P}<0.001)$, situación que los autores asociaron con una mayor incidencia de desorden de personalidad antisocial y abuso de sustancias. ${ }^{13}$ Así, se tiene en cuenta que los factores siquiátricos y culturales también podrían tener una gran influencia sobre la violencia juvenil, constituyéndose entonces como un sesgo de este estudio, puesto que dentro del registro utilizado para la obtención de datos, no se incluyen información de evaluaciones psiquiátricas.

\section{CONCLUSION}

En la población entre 14-26 años las lesiones por trauma entre los años 2012-2014 se presentó una disminución significativa en la incidencia anual $(p<0.0001)$ y disminución en la tasa de mortalidad global. Al parecer la tendencia observada puede ser el reflejo del impacto de la implementación de la política pública.

\section{REFERENCIAS}

1. Organización Mundial de la Salud. Informe mundial sobre la violencia y salud: sinopsis. OMS 2002. [Internet]. Consultado en: julio 25 2016. Disponible en: http://apps.who.int/iris/ bitstream/10665/67411/1/a77102_spa.pdf

2. Organización Mundial de la Salud. Los jóvenes y los riesgos sanitarios: Informa de la secretaría. 64. ${ }^{\text {a }}$ ASAMBLEA MUNDIAL DE LA SALUD. 2011. Consultado en: Julio 25 de 2016. Disponible en: http://apps.who.int/gb/ebwha/ pdf_files/WHA64/A64_25-sp.pdf

3. Mercy J, Butchart A, Farrington D, Magdalena C. Youth violence. World report on violence and health. In: Krug EG, Dahlberg LL, Mercy JA, Zwi AB, Lozano R., editors. World report on violence and health. Geneva: World Health Organization; 2002.

4. Centers for Disease Control and Prevention, National Center for Injury Prevention and Control. Web-based Injury Statistics Query and Reporting System (WISQARS) [online]. (2010). [cited 2012 Oct 19] Available from www.cdc.gov/injury.

5. Organización Mundial de la Salud, Oficina de las Naciones Unidas Contra la Droga y el Delito. Informe sobre la situación mundial de la prevención de la violencia 2014. OMS, UNODC. 2014. [Consultado: Octubre 2016] Disponible en: http://apps.who.int/iris/bitstream/10665/145089/1/ WHO_NMH_NVI_14.2_spa.pdf?ua $=1$.

6. Chan LS, Kipke MD, Schneir A, Iverson E, Warf C, Limbos MA, Shekelle P. Preventing violence and related health-risking social behaviors in adolescents. Evidence report. AHRQ Publication No 04-E032-2, 2004.

7. Banco Mundial. Criminalidad, violencia y su impactante lastre para el desarrollo de América Latina. 2011. [Consultado: Septiembre 2016]. Disponible en: http://www.bancomundial. org/es/news/press-release/2011/04/07/crime-violencestaggering-toll-central-american-development.

8. Instituto Nacional de Medicina Legal y Ciencias Forenses. Forensis 2012: Datos para la vida. Grupo Centro de Referencia Nacional sobre Violencia. 2012. [Consultado: Agosto 2016]. Disponible en: http://www.medicinalegal.gov.co/document s/10180/34861/3+2+violencia+interpersonal+forensis+2012. pdf/b6a82964-a6dc-4568-b715-0dd6712d5054.

9. Ordóñez CA, Pino LF, Tejada JW, Badiel M, Loaiza JH, Mata LV, Aboutanos MB. Experience of two first level hospitals in the southwest region of Colombia on the implementation of the Panamerican Trauma Society International Trauma Registry. Rev Col Bras Cir 2012;39(4), 255-262.

10. Organización Mundial de la Salud. Violencia Juvenil. Notas descriptivas. 2016

11. Julio Jacobo Waiselfisz. Mapa da violência 2015. Adolescentes de 16 e 17 anos do Brasil. FLACSO Brasil. Rio de Janeiro, 2015.

12. Yu R, Aaltonen M, Branje S, Ristikari T, Meeus W, SalmelaAro K, Goodwin GM, Fazel S. (2017). Depression and violence in adolescence and young adults: findings from three longitudinal cohorts. J Am Acad Child Adolesc Psychiatry 2017;56(8):652-658.

13. Coid J, Hu J, Kallis C, Ping Y, Zhang J, Hu Y, Bui L, Ullrich S, Bebbington P. A cross-national comparison of violence among young men in China and the UK: psychiatric and cultural explanations. Soc Psychiatry Psychiatr Epidemiol 2017;52(10):1267-1279. 
Based on registry data, Ms Reyes and associates report from Cali that the implementation of local youth plan between 2012 and 2014 to reduce youth violence had a positive impact on the population, especially between the ages of 14 and 26 years. There was a decrease in the indicators of violence. The authors themselves point out that other studies documented that large failures were found in the implementation of youth plans. They also, to their credit, admit that youth violence is multifactorial (e.g., psychiatric issues, substance addiction). The positive results noted in the study, therefore, may have nothing to do with public policy implementation.

Despite these comments, this is an excellent example at registry data collection, analysis, and concept generation that can lead to further refinement of the problem. The value of the trauma registry, as is being shown so well by the Cali group, should be an important lesson for other trauma centers.

Finally, the principal author, a medical student, deserves much credit and acclaim for her initiative and work.

Rao R Ivatury

Editor-in-Chief. Panamerican Journal of Trauma Critical Care \& Emergency Surgery 